\title{
Gray Scale Structuring Element Decomposition
}

\author{
Octavia Camps \\ Dept. of Electrical and Computer Engineering, \\ The Pennsylvania State University, \\ University Park, PA 16802
}

\author{
Robert Haralick and Tapas Kanungo \\ Department of Electrical Engineering, FT-10 \\ University of Washington \\ Seattle WA 98195
}

\begin{abstract}
Efficient implementation of morphological operations requires the decomposition of structuring elements into the dilation of smaller structuring elements. Zhuang and Haralick presented an algorithm to find optimal decompositions of structuring elements in binary morphology. In this paper we extend the algorithm to find optimal structuring element decomposition for gray scale morphology.
\end{abstract}

\section{Introduction}

When the structuring element used in a morphological operation is larger than the largest element that the hardware can handle in one stage, the structuring element must be decomposed into smaller structuring elements. Each of these elements has to be such that the hardware is capable of handling it and whose morphological composition is the given structuring element.

An algorithm that finds an optimal decomposition for binary structuring elements was proposed in [1]. All binary morphological operations are naturally extended to gray scale imagery by using the Top and Umbra operations. In this paper we use this idea to extend the algorithm proposed in [1] to be used in gray scale morphology.

\section{Statement of the problem}

The gray scale structuring element decomposition is formally stated as:

Given a structuring element $s(x)$, find the smallest integer $M$ and the corresponding structuring elements $h_{1}(x), h_{2}(x), \ldots, h_{M}(x)$ such that

$$
s(x)=\left(h_{1} \oplus h_{2} \oplus \cdots \oplus h_{M}\right)(x)
$$

where each $h_{i}(x)$ satisfies the hardware requirements.

In this paper we will consider that the structuring element $s$ is a discrete function defined in a finite domain.

\section{Notation and definitions}

\subsection{Gray scale morphology}

For completeness we begin by stating some definitions and theorems. An extended presentation of the definitions and the proof of the theorems in this subsection can be found in Haralick et al. [2].
The four binary morphological operations of dilation, erosion, opening and closing are all extended to gray scale morphology by introducing the concept of the top surface of a set and the related concept of the umbra of a surface.

Def. 3.1 Let $A \subseteq E^{N}$ and $F=\left\{x \in E^{N-1} \mid\right.$ for some $y \in E,(x, y) \in A\}$. The top or top surface of $A$, denoted by $T[A]: F \rightarrow E$, is defined by

$$
T[A](x)=\max \{y \mid(x, y) \in A\} .
$$

Def. 3.2 A set $A \subseteq E^{N-1} \times E$ is an umbra if and only if $(x, y) \in A$ implies that $(x, z) \in A$ for every $z \leq y$.

Def. 3.3 Let $F \subset E^{N-1}$ and $f: F \rightarrow E$. The umbra of $f$, denoted by $\bar{U}[f], U[f] \subseteq F \times E$, is defined by

$$
U[f]=\{(x, y) \in F \times E \mid y \leq f(x)\} .
$$

The top of the umbra of a function $f$ is the function $f$ itself:

Theorem 3.1 Let $F \subseteq E^{N-1}$ and $f: F \rightarrow E$. Then $T[U[f]]=f$.

Having defined the operations of taking the Top set of a set and the Umbra of a surface, gray scale dilation and erosion can be defined:

Def. 3.4 Let $F, K \subset E^{N-1}$ and $f: F \rightarrow E$ and $k: K \rightarrow E$. The dilation of $f$ by $k$ is denoted by $f \oplus k, \vec{f} \oplus \vec{k}: F \oplus K \rightarrow E$, and is defined by

$$
f \oplus k=T[U[f] \oplus U[k]] .
$$

Def. 3.5 Let $F, K \subseteq E^{N-1}$ and $f: F \rightarrow E$ and $k: K \rightarrow E$. The erosion of $f$ by $k$ is denoted by $f \ominus k, f \ominus k: F \ominus K \rightarrow E$, and is defined by

$$
f \ominus k=T[U[f] \ominus U[k]] .
$$

Gray scale dilation and erosion can be accomplished by using maximum and minimum operations. 
Theorem 3.2 Let $f: F \rightarrow E$ and $k: K \rightarrow E$. Then $f \oplus k: F \oplus K \rightarrow E$ can be computed by

$(f \oplus k)(x)=\max \{f(x-z)+k(z) \mid x-z \in F$ and $z \in K\}$.

Theorem 3.3 Let $f: F \rightarrow E$ and $k: K \rightarrow E$. Then $f \ominus k: F \ominus K \rightarrow E$ can be computed by

$$
(f \ominus k)(x)=\min \{f(x+z)-k(z) \mid z \in K\} .
$$

Gray scale opening and closing are defined in an analogous way to opening and closing in the binary morphology:

Def. 3.6 Let $f: F \rightarrow E$ and $k: K \rightarrow E$. The opening of $f$ by the structuring element $k$ is denoted by $f \circ k$ and is defined by

$$
f \circ k=(f \ominus k) \oplus k .
$$

Def. 3.7 Let $f: F \rightarrow E$ and $k: K \rightarrow E$. The closing of $f$ by the structuring element $k$ is denoted by $f \bullet k$ and is defined by

$$
f \bullet k=(f \oplus k) \ominus k .
$$

\subsection{Translation in gray scale morphology}

To solve the decomposition problem we need to define the translation of a gray scale structuring element. In this subsection we define the translation of a structuring element by a point and derive some useful properties.

Def. 3.8 Let $F \subseteq E^{N-1}$ and $f: F \rightarrow E$. The translation of $f$ by $(\alpha, \beta)$ with $\alpha \in E^{N-1}$ and $\beta \in E$, is denoted by $(f)_{(\alpha, \beta)}$ and is defined by

$$
(f)_{(\alpha, \beta)}(x)=f(x-\alpha)+\beta
$$

for every $x \in F$.

Given $F \subseteq E^{N-1}, f: F \rightarrow E$ and $(\alpha, \beta) \in E^{N}$. The gray scale translation has the following properties.

Theorem 3.4 $(U[f])_{(\alpha, \beta)}=U\left[f_{(\alpha, \beta)}\right]$

Proof: Let $(x, y) \in E^{N}$. The point $(x, y) \in(U[f])_{(\alpha, \beta)}$ if and only if there exists $(u, v) \in U[f]$ such that $(x, y)=(u, v)+(\alpha, \beta)$.

By definition of umbra $(u, v) \in U[f]$ if and only if $v \leq f(u)$. Then, $y=v+\beta \leq f(u)+\beta=f(x-\alpha)+\beta$. Hence, $(x, y) \in U\left[f_{(\alpha, \beta)}\right]$.

Theorem 3.5 $T\left[(U[f])_{(\alpha, \beta)}\right]=(T[U[f]])_{(\alpha, \beta)}$

Proof: Let $x \in E^{N-1}$. Then by theorem 3.4, $T\left[(U[f])_{(\alpha, \beta)}\right](x)=T\left[U\left[f_{(\alpha, \beta)}\right]\right](x)=f_{(\alpha, \beta)}(x)=$ $(T[U[f]])_{(\alpha, \beta)}(x) . \diamond$

The translation of a function can be expressed as the dilation of the given function and another function given by the translation point.
Theorem 3.6 There exists $G \subseteq E^{N-1}, g: G \rightarrow E$ such that $f_{(\alpha, \beta)}=f \oplus g$.

Proof: Let $G=\alpha$ and $g(\alpha)=\beta$. By definition 4, $f \oplus g=T[U[f] \oplus U[g]]$.

A point $(x, y) \in U[f] \oplus U[g]$ if and only if there exists $\left(x_{1}, y_{1}\right) \in U[f]$ and $\left(x_{2}, y_{2}\right) \in U[g]$ such that $(x, y)=\left(x_{1}, y_{1}\right)+\left(x_{2}, y_{2}\right)$.

But, $(x, y) \in U[f]$ implies $y \leq f(x)$ and $\left(x_{2}, y_{2}\right) \in$ $U[g]$ implies $x_{2}=\alpha$ and $y_{2} \leq g\left(x_{2}\right)=\beta$.

Then, $y=y_{1}+y_{2} \leq f\left(x_{1}\right)+\beta=f(x-\alpha)+\beta$. Hence, by definition of Top, $(f \oplus g)(x)=\max \{z \mid z \leq$ $f(x-\alpha)+\beta\}=f(x-\alpha)+\beta=f_{(\alpha, \beta)} \cdot \diamond$

The dilation and erosion operations commute with translation.

Theorem 3.7 Let $f_{1}: E^{N-1} \rightarrow E$ and $f_{2}: E^{N-1} \rightarrow$ $E$. Then, $f_{1(\alpha, \beta)} \oplus f_{2}=\left(f_{1} \oplus f_{2}\right)_{(\alpha, \beta)}$.

Proof: By definition of dilation and by theorem 3.5 $f_{1(\alpha, \beta)} \oplus f_{2}=T\left[U\left[f_{1(\alpha, \beta)}\right] \oplus U\left[f_{2}\right]\right]=T\left[\left(U\left[f_{1}\right] \oplus\right.\right.$ $\left.\left.U\left[f_{2}\right]\right)_{(\alpha, \beta)}\right]=\left(T\left[U\left[f_{1}\right] \oplus U\left[f_{2}\right]\right]\right)_{(\alpha, \beta)}=\left(f_{1} \oplus\right.$ $\left.f_{2}\right)_{(\alpha, \beta)} . \diamond$

Theorem 3.8 If $F$ is finite, then $f_{(\alpha, \beta)} \ominus f=g(x)$ with $g: G=\alpha \rightarrow E$ and $g(\alpha)=\beta$.

Proof: By definition of erosion and by theorem 3.5, $f_{(\alpha, \beta)} \ominus f=T\left[U\left[f_{(\alpha, \beta)}\right] \ominus U[f]\right]=T\left[(U[f])_{(\alpha, \beta)} \ominus\right.$ $U[f]=T\left[(U[f] \ominus U[f])_{(\alpha, \beta)}\right]$.

A point $(x, y) \in U[f] \Theta U[f]$ if and only if for every $(u, v) \in U[f],(x, y)+(u, v) \in U[f]$. But, $(x, y)+$ $(u, v) \in U[f]$ if and only if $x+u \in F$ and $y+v \leq$ $f(x+u)$.

If $F$ is finite, then there exists $X_{1}$ and $X_{2}$ such that $X_{1} \leq x \leq X_{2}$ for every $x \in F$

Let $u=X_{1}$. Then, $x+X_{1} \in F$ implies $x \geq 0$. Now let $u=X_{2}$. Then, $u+X_{2} \in F$ implies $x \leq \overline{0}$. Hence, $x$ must be 0 .

Consider $(u, v)=\left(X_{1}, f\left(X_{1}\right)\right) \in U[f]$. But, $y+v=$ $y+f\left(X_{1}\right) \leq f\left(X_{1}\right) \Rightarrow y \leq 0$.

Now, $f_{(\alpha, \beta)} \ominus f=T\left[(\{(x, y) \mid x=0, y \leq 0\})_{(\alpha, \beta)}\right]=$ $T[\{(\boldsymbol{x}, y) \mid x=\alpha, y \leq \beta\}]=(\alpha, \beta) \diamond$

\section{Reduction of the problem.}

The problem we solve in this section is to construct a decomposition of $s$, into $h_{1}, h_{2}, \ldots, h_{M}$, having the smallest $M$, if one exists, where each $h_{i}$ has no more points than the prescribed fixed number $k$ determined by hardware constraints. We also assume that the domain of $s$ is finite.

To determine such a decomposition of $s$, if one exists, requires a combinatorial search process for the domains of the functions $h_{i}$ and for the values of these functions at each point of the domain. Our algorithm limits the possible domains and the possible values of the functions $h_{i}$, and thereby greatly reduces the search space.

We will show that the gray scale structuring element decomposition is equivalent to one with fewer degrees of freedom: 
Theorem 4.1 If $s=h_{1} \oplus h_{2} \oplus \cdots \oplus h_{M}$, then there exists $(\alpha, \beta)$ and $j_{1}, j_{2}, \ldots, j_{M}$ such that:

1. \#j $j_{i}=\# h_{i}$, for $i=1, \ldots, M$

2. $j_{i}(0)=0$ and $j_{i}(x) \leq 0$, for $i=1, \ldots, M$

3. $s=\{(\alpha, \beta)\} \oplus j_{1} \oplus j_{2} \oplus \cdots \oplus j_{M}$

4. $\{(\alpha, \beta)\}=s \ominus\left\{j_{1} \oplus j_{2} \oplus \cdots \oplus j_{M}\right\}$

Proof: Let $\alpha_{i}$ be such that $h_{i}\left(\alpha_{i}\right)=\beta_{i}$ and $h_{i}(x) \leq \beta_{i}$ for every $x$ in the domain of $h_{i}$.

Consider $j_{i}=\left(h_{i}\right)_{(-\alpha,-\beta)}$. Then, $j_{i}(0)=h_{i}\left(\alpha_{i}\right)-$ $\beta_{i}=0$ and $j_{i}(x)<0$ for every $x$ in the domain of $j_{i}$. Note that with this definition $h_{i}$ and $j_{i}$ have the same number of points.

Next we will show that $s=\{(\alpha, \beta)\} \oplus j_{1} \oplus j_{2} \oplus$ $\cdots \oplus j_{M}$. Since $j_{i}=\left(h_{i}\right)_{\left(-\alpha_{i},-\beta_{i}\right)}$, by the definition of translation, we have that $h_{i}=\left(j_{i}\right)_{\left(\alpha_{i}, \beta_{i}\right)}$. Therefore,

$s=h_{1} \oplus h_{2} \oplus \cdots \oplus h_{M}=\left(\left(j_{1}\right)_{\left(\alpha_{1}, \beta_{1}\right)} \oplus \cdots\left(j_{M}\right)_{\left(\alpha_{M}, \beta_{M}\right)}\right)$

By theorem 3.7,

$$
s=\left(j_{1} \oplus j_{2} \oplus \cdots \oplus j_{M}\right)_{(\alpha, \beta)}
$$

where

$$
(\alpha, \beta)=\left(\alpha_{1}+\alpha_{2}+\cdots+\alpha_{M}, \beta_{1}+\beta_{2}+\cdots+\beta_{M}\right) .
$$

Then, by theorem $3.6, s$ can be expressed as the dilation $s=\{(\alpha, \beta)\} \oplus\left(j_{1} \oplus j_{2} \oplus \cdots \oplus j_{M}\right)$. Furthermore, by theorem $3.8\{(\alpha, \beta)\}=s \ominus\left(j_{1} \oplus j_{2} \oplus \cdots \oplus j_{M}\right)$. $\diamond$

Theorem 4.1 in effect shows that the original problem can be reduced to one with fewer degrees of freedom. Since $j_{i}(0)=0$, there is one less unknown point to determine each $j_{i}$ compared to the number of unknown points in the corresponding $h_{i}$. Furthermore, the extra unknown $(\alpha, \beta)$ is determined without searching, once all the $j_{i}$ have been determined.

The search for the decomposition elements $j_{i}$ can be reduced by establishing that if $s=\{(\alpha, \beta)\} \oplus j_{1} \oplus$ $j_{2} \cdots \oplus j_{M}$, with $j_{i}(0)=0$ and $j_{i}(x) \leq 0$, then there exists $x_{1}$ and $x_{2}$ in the domain of $s$ such that $x=$ $x_{1}-x_{2}$ and $s\left(x_{1}\right)-s\left(x_{2}\right) \leq j_{i}(x) \leq 0$. The proof consists of various steps given by theorems 4.2 to 4.6 .

Theorem 4.2 Let $f_{1}: F_{1} \rightarrow E$ and $f_{2}: F_{2} \rightarrow E$ Then, for every $x \in F_{1} \oplus F_{2}$ there exists $x_{1} \in F_{1}$ and $x_{2} \in F_{2}$ such that $x=x_{1}+x_{2}$ and $\left(f_{1} \oplus f_{2}\right)(x)=$ $f_{1}\left(x_{1}\right)+f_{2}\left(x_{2}\right)$.

Proof: By theorem 3.2, $\left(f_{1} \oplus f_{2}\right)(x)=\max \left\{f_{1}(x-\right.$ $u)+f_{2}(u) \mid x-u \in F_{1}$ and $\left.u \in F_{2}\right\}$.

Let $u_{1}$ be such that $f_{1}\left(x-u_{1}\right)+f_{2}\left(u_{1}\right)$ is maximum Then there exists $x_{1}=x-u_{1} \in F_{1}$ and $x_{2}=u_{1} \in F_{2}$ such that $x=x_{1}+x_{2}$ and $\left(f_{1} \oplus f_{2}\right)(x)=f_{1}\left(x_{1}\right)+$ $f_{2}\left(x_{2}\right)$.

Theorem 4.3 Let $A \subseteq E^{N-1}$ and $B \subseteq E^{N-1}$. Then, $A \circ B \subseteq A$.
Proof: Let $a \in A \circ B$. Then there exists an $x \in A \ominus B$ and $b \in B$ such that $a=x+b$. But $x \in A \ominus B$ implies that for every $y \in B, x+y \in A$. Since $b \in B, x+b \in A$. But $a=x+b$ so that $a \in A$. $\diamond$

Theorem 4.4 Let $A=B \oplus C$. Then, $A \circ C=A$.

Proof: Let $a \in A$. Since $A=B \oplus C$, there exists a $b \in B$ and a $c \in C$ such that $a=b+c$. But for every $x \in B$ and $c \in C, x+c \in A$. Since $b \in B$, we must have for every $y \in C, b+y \in A$. This implies $b \in A \ominus C$ and $b \in B$ imply $a \in(A \ominus C) \oplus C$. Hence $A \subseteq(A \circ C)$. By theorem 4.3, $A \circ C \subseteq A$. Thus, $A \circ C=A . \diamond$

Theorem 4.5 Let $s=a \oplus j$. Then, $s=s \circ j$.

Proof: By definition of dilation, $s(x)=T[U[a] \oplus$ $U[j]](x)$. Taking the umbra at both sides, $U[s]=$

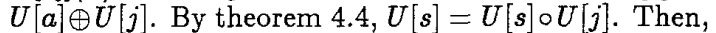
$s(\boldsymbol{x})=T[U[s] \circ U[j]](x)=(s \circ j)(x) \diamond \diamond$

Theorem 4.6 Let $s=s \circ j, j(0)=0$ and $j(x) \leq 0$. Then, there exists $x_{1}$ and $x_{2}$ in the domain of $s$ such that $x=x_{1}-x_{2}$ and $j(x) \geq s\left(x_{1}\right)-s\left(x_{2}\right)$.

Proof: Since $s=(s \ominus j) \oplus j$, by theorem 4.2, for every $x$ in the domain of $j$ there exists $x_{1}$ and $x_{2}$ in the domain of $s$ such that $x=x_{1}-x_{2}$ and $s\left(x_{1}\right)=(s \ominus j)\left(x_{2}\right)+$ $j(x)$. But by theorem 3.3, $(s \ominus j)(y)=\min \{s(y+$ $u)-j(u) \mid u \in J\} \leq s(y)$ for every $y \in S \ominus J$, since $j(0)=0$ and $j(x) \leq 0$. Then, $j(x) \geq s\left(x_{1}\right)-s\left(x_{2}\right)$ and $x=x_{1}-x_{2} . \diamond$

We have shown that the possible domains of the functions $j_{i}$ are those formed by differences between points of the domain of $s$. Furthermore, we showed that $s\left(x_{1}\right)-s\left(x_{2}\right) \leq j_{i}\left(x_{1}-x_{2}\right) \leq 0$ for each point.

\subsection{Search.}

In this section we give a complete description of the tree search using the elements developed in the previous section. The algorithm is a generalization of the algorithm proposed in [1].

Suppose that the structuring element $s$ has $m$ points and that each $j_{i}$ must have $k$ points one of which is $(0,0)$.

To reduce the tree search we take into account the following two considerations:

I) Since dilation is commutative, for a node associated with $j_{q}$ there is only need to consider the possible descendents $j_{q+1}, j_{q+2}, \ldots, j_{J}$.

II) Forward checking can be used to control the growth of the tree. We will show that if at some level $l, j_{1}, j_{2}, \ldots, j_{l}$ have been determined, then the only $j$ 's that need to be considered for any node in the subtree below are those that satisfy:

$$
\left(t_{l} \ominus j\right) \oplus j \oplus k_{l}=s
$$

where $t_{l}=s \ominus k_{l}$, and $k_{l}=j_{1} \oplus j_{2} \oplus \cdots \oplus j_{l}$.

Assume that $s=\{(\alpha, \beta)\} \oplus j_{1} \oplus \cdots \oplus j_{M}$ with $M>l$, and we need to find $j_{l+1}$. The structuring element $s$ can be rewritten as:

$$
s=\left(\left\{(\alpha, \beta) \oplus j_{l+2} \oplus \cdots \oplus j_{M}\right) \oplus k_{l} \oplus j_{l+1}\right.
$$


Then, by theorem $4.5, s$ must be open under $k_{l}$ dilated by the function $j$, the candidate to be selected as $j_{l+1}$ :

$$
s=\left(s \ominus\left(k_{l} \oplus j\right)\right) \oplus\left(k_{l} \oplus j\right)=\left(t_{l} \ominus j\right) \oplus j \oplus k_{l}
$$

It is also true, that this condition holds for the level 1 considered and for any node in the subtree below: Let $m>l$. Then, $j$ to be considered as a child of a node at level $m$ must satisfy: $s=s \circ\left(k_{m} \oplus j\right)$, but, $k_{m} \oplus j=\left(k_{l} \oplus j\right) \oplus\left(j_{l+1} \oplus j_{l+2} \oplus \cdots \oplus j_{M}\right)$.

Theorem 4.7 If $S=S \circ(A \oplus B)$ then $S=S \circ A$.

Proof: See Haralick et al. [2].

Theorem 4.8 If $s=s \circ(a \oplus b)$ then $s=s \circ a$.

Proof: By hypothesis, $s(x)=T[U[s] \circ U[a \oplus b]](x)$. Then, by theorem $4.7, U[s]=U[s] \circ(U[a] \oplus U[b])=$ $U[s] \circ U[a]$. Therefore, $s(x)=T[U[s] \circ U[a](x)$ and $s(x)=\left(\begin{array}{lll}s & \circ a\end{array}\right)(x) \diamond$

Theorem 4.8 shows that if $s$ is not open under $k_{l} \oplus j$, then it is not open under $k_{m} \oplus j$. This condition can be used as a forward check to prune the search tree.

\subsection{Algorithm}

The algorithm to accomplish the decomposition of a gray scale structuring element $s$ consists of a breadth first tree search with forward checking.

A node $i$ in the tree corresponds to a candidate structuring element, $j_{i}(x)$. Each node $i$ has also associated with it the following entities:

1. A list of all its possible descendents, $L_{i}$.

2. The partial decomposition so far, $k_{i}=j_{1} \oplus$ $j_{2} \cdots \oplus j_{i}$, corresponding to its dilation with all its predecessor nodes.

3. The undecomposed part or residue, $t_{i}=s \in k_{i}$.

The root of the tree is initialized such that $L_{0}=$ $j_{1}, j_{2}, \ldots, j_{M}$ is the set of all possible structuring elements, $k_{o}=\{0\}$, and $t_{o}=s$. Before opening a node in the tree a forward check is made through the possible descendents of the node. The checking eliminates those structuring elements in $L_{i}$ that do not satisfy $s=\left(t_{i} \ominus j\right) \oplus\left(j \ominus k_{i}\right)$. Once the forward checking is finished, the nodes corresponding to the elements in $L_{i}$ that survived the test are opened.

Any decomposition found at the lower level of the tree is an optimal decomposition. A decomposition is found when the number of elements in the domain of the residue $t$ is one, and its functional value $t(\alpha)=\beta$ corresponds to the $(\alpha, \beta)$ appearing in the decomposition $s=\{(\alpha, \beta)\} \oplus j_{1} \oplus \cdots \oplus j_{M}$.

\section{Example}

In the following we give an example illustrating the use of the algorithm to decompose an structuring element into sets of two points. Let $s(x)$ be the gray scale structuring element to be decomposed, as shown in figure 1 . There are thirty five candidate structuring elements, defined by $j_{i}(0)=0 ; j_{i}\left(x_{i}\right)=y$, with $0 \leq y \leq s\left(x_{1}\right)-s\left(x_{2}\right)$, and $x_{i}=x_{1}-x_{2}$. However,

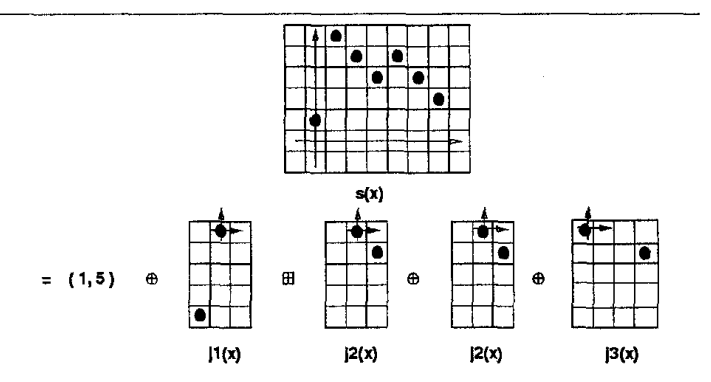

Figure 1: A decomposition of a gray scale structuring element.

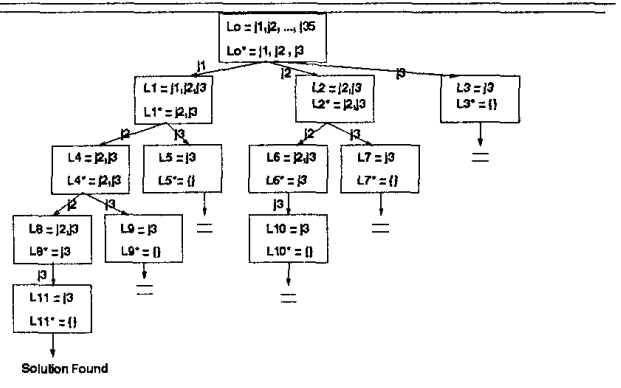

Figure 2: Illustrates the tree search for $s(x)$.

only three of the candidates survive the forward check: $\left\{j_{1}(0)=0 ; j_{1}(-1)=-4\right\},\left\{j_{2}(0)=0 ; j_{2}(1)=-1\right\}$, and $\left\{j_{3}(0)=0 ; j_{3}(3)=-1\right\}$. Figure 2 shows the tree created by the algorithm. The optimal decomposition of $s, s=\{(1,5)\} \oplus j_{1}(x) \oplus j_{2} \oplus j_{2} \oplus j_{3}$ is shown in figure 1.

\section{Conclusion}

The gray scale structuring element decomposition problem can be solved in a similar way to the binary structuring element decomposition problem. In this paper, we showed that the decomposition problem can be solved by simply searching among a finite set of values. The essence of the algorithm is the same as the essence of the binary problem: (1) the domain of the structuring elements participating in the decomposition must have members which are the differences between members of the domain of the given structuring element and (2) that it is necessary for the undecomposed part of the structuring element to be morphologically open with respect to any structuring element participating in its further decomposition.

\section{References}

1. Zhuang, Xinhua and Haralick, Robert (1986) Morphological Structuring Element Decomposition. CVGIP 35, 370-382.

2. Haralick, Robert, Sternberg Stanley and Zhuang, Xinhua (1987) Image Analysis Using Mathematical Morphology. IEEE Transactions on Pattern Analysis and Machine In telligence, vol. PAMI-9, no.4, pp. 532-550, july 1987. 